\title{
Period of Care, Encounter-Based
}

National Cancer Institute

\section{Source}

National Cancer Institute. Period of Care, Encounter-Based. NCI Thesaurus. Code C154530.

A time period of care where the start and stop dates are populated from the earliest observed encounter and latest observed encounter. 